\title{
Characterization of new microsatellite loci for population genetic studies in the Smooth Cauliflower Coral (Stylophora sp.)
}

\author{
Eulalia Banguera-Hinestroza $\cdot$ Pablo Saenz-Agudelo $\cdot$ \\ Till Bayer · Michael L. Berumen · Christian R. Voolstra
}

Received: 18 December 2012/ Accepted: 21 December 2012/Published online: 9 January 2013

(C) The Author(s) 2013. This article is published with open access at Springerlink.com

\begin{abstract}
A total of one hundred microsatellites loci were selected from the draft genome of Stylophora pistillata and evaluated in previously characterized samples of Stylophora cf pistillata from the Red Sea. 17 loci were amplified successfully and tested in 24 individuals from samples belonging to a single population from the central region of the Red Sea. The number of alleles ranged from 3 to 15 alleles per locus, while observed heterozygosity ranged from 0.292 to 0.95 . Six of these loci showed significant deviations from Hardy-Weinberg equilibrium (HWE) expectations, and 4/136 paired loci comparisons suggested linkage disequilibrium after Bonferroni corrections. After excluding loci with significant HWE deviation and evidence of null alleles, average genetic diversity over loci in the population studied $(\mathrm{N}=24$, Nloci $=11)$ was $0.701 \pm 0.380$. This indicates that these loci can be used effectively to evaluate genetic diversity and undertake population genetics studies in Stylophora sp. populations.
\end{abstract}

Keywords Stylophora - Tetra and dinucleotide microsatellites $\cdot$ Red Sea $\cdot$ Coral

Reef building corals of the genus Stylophora (Schweigger 1819) have a widespread distribution in the Indo-pacific with the highest diversity being recorded in the Indian Ocean and

E. Banguera-Hinestroza - P. Saenz-Agudelo - T. Bayer ·

M. L. Berumen · C. R. Voolstra $(\square)$

Red Sea Research Center, King Abdullah University of Science and Technology, Thuwal 23955-6900, Saudi Arabia

e-mail: christian.voolstra@kaust.edu.sa

M. L. Berumen

Biology Department, Woods Hole Oceanographic Institution,

Woods Hole, MA 02543, USA the Red Sea (Veron 2000). However, it is unclear whether this diversity is due to the presence of several cryptic species in the region or simply a reflection of well-differentiated populations with high phenotypic plasticity. The lack of taxonomic and molecular information in this regard has led to confusion in terms of the real delineation between populations and species in this group (see Obura 2012). Recent studies have raised important and controversial questions about the delineation of species within the genus and highlighted the need for a clear assessment of the levels of population differentiation within cryptic species (i.e. Flot et al. 2011; Stefani et al. 2011).

In contrast to other well-studied taxa in the region (see Carpenter et al. 2011), phylogeographic and population genetics studies in species of the genus Stylophora are scarce. Only a few studies have investigated the level of genetic diversity within and among closely related populations (Ayre and Hughes 2000; Nishikawa et al. 2003; Zvuloni et al. 2008; Douek et al. 2011) and only one has investigated genetic connectivity at local and interoceanic spatial scales (Takabayashi et al. 2003). Here, we developed a panel of 17 specific microsatellites loci for population and phylogeographic studies in Stylophora spp. We expect that these markers will contribute to a better assessment of population structure, connectivity, and evolutionary history of these species throughout their range. In the long term, their use will provide a better understanding of the evolutionary mechanisms promoting the long-term survival of coral reefs populations, identify vulnerable populations, and promote conservation policies.

Di- and tretranucleotide repeats were identified in the draft genome of Stylophora pistillata (Voolstra and Aranda, unpublished data) using the software Tandem Repeats Finder (Benson 1999) and primers flanking suitable repeats were designed using the software Primer3 
(Rozen and Skaletsky 2000). A total of one hundred loci were tested for positive amplification and polymorphism in 24 individuals from a single population from Shi'b Nazar reef, central Red Sea $\left(22^{\circ} 19.142^{\prime} \mathrm{N}, 38^{\circ} 51.263^{\prime} \mathrm{E}\right)$. These individuals were morphologically identified as $S$. pistillata although subsequent mtDNA analyses showed that they cluster together in a single clade, well-differentiated from those previously reported by other studies (see Flot et al. 2011). For the purpose of this study we will subsequently refer to our samples as coming from Stylophora cf pistillata.

Approximately $1 \mathrm{~cm}$ of each coral colony was disrupted using a TissueLyser II (Qiagen) in presence of lysis buffer (Qiagen) to release high-quality DNA. All DNA extractions were performed following the protocols from Qiagen DNeasy Plant Mini Kit (Qiagen). Multiplex PCRs were performed using 20-50 ng of DNA template using the Qiagen Multiplex PCR kit according to the manufacturer's recommendations. The final volume for all reactions was fixed at $10 \mu \mathrm{l}$, final concentration of primers was set at $0.1 \mu \mathrm{M}$, and primers mixes were selected according to the expected sizes (Table 1). Samples were amplified using the following thermal cycle profile: A first denaturation step at $95{ }^{\circ} \mathrm{C}$ for $15 \mathrm{~min}$ (for hot start Taq DNA polymerase, Qiagen), followed by 25 cycles at $94{ }^{\circ} \mathrm{C}$ for $30 \mathrm{~s}$, annealing at a locus-specific temperature $\left(60{ }^{\circ} \mathrm{C}\right.$ for all primer mixes) for $90 \mathrm{~s}$, and an extension at $72{ }^{\circ} \mathrm{C}$ for $60 \mathrm{~s}$. The final extension was set at $60{ }^{\circ} \mathrm{C}$ for $30 \mathrm{~min}$. Screening was performed using fluorescentlabeled primers (6-FAM, PET, VIC and NED, Table 1), fragment sizes were analyzed using an Applied
Biosystems 3730 XL genetic analyzer, and allele sizes were scored using GeneMapper ${ }^{\circledR} 4.0$ software (Applied Biosystems).

After scoring the alleles, the program MicroChecker v2.2.3 (Van Oosterhout et al. 2004) was used to test for the presence of null alleles and error due to stuttering. Departure from Hardy-Weinberg Equilibrium (HWE) and linkage disequilibrium were evaluated using Arlequin ver 3.5 (Excoffier and Lischer 2010) and basic statistics were estimated using Genalex v6.5 (Peakall and Smouse 2012).

In the sample analyzed $(\mathrm{N}=24)$, seventeen microsatellite loci showed moderate-to-high levels of polymorphism; the number of alleles ranged from 3 to 15 . Observed heterozygosity ranged from 0.292 to 0.95 , while expected heterozygosity ranged from 0.49 to 0.891 . Six loci showed significant deviation from HWE expectations (alpha = 0.05 ), and 4 of them showed significant deviations after Bonferroni corrections (Stylo_26, Stylo_39, Stylo_72, Stylo_95; $P<0.001)$. Results from Micro Checker suggested the presence of null alleles for all six loci. Linkage disequilibrium was significant in 4/136 paired loci comparisons after Bonferroni correction (Table 2). After excluding loci with significant HWE deviation and evidence of null alleles, the average genetic diversity over loci in this population was $0.701 \pm 0.380$, while the mean observed and expected heterozygosities were $0.645 \pm 0.159$ and $0.681 \pm 0.124$ respectively. Ongoing studies will use these new markers in conjunction with mitochondrial markers to determine the degree of genetic differentiation and level of genetic flow on small and large scales from a North-South gradient in the Red Sea.

Table 1 Primer sequences and general description of 17 new microsatellites loci for population and phylogeographic studies in Stylophora sp.

\begin{tabular}{|c|c|c|c|c|c|c|}
\hline Locus & Motif & Label & Forward primer $\left(5^{\prime}-3^{\prime}\right)$ & Reverse primer $\left(5^{\prime}-3^{\prime}\right)$ & Size range & $\mathrm{N}_{\mathrm{A}}$ \\
\hline Stylo_17 & $(\mathrm{TA})_{10}$ & 6-FAM & CAAGTATTTTCACAGGGCGG & TCAAAACCTGAAGCAATCCC & $161-179$ & 8 \\
\hline Stylo_19 & $(\mathrm{AG})_{11}$ & PET & ATCTTTCGTTCTCACGACGG & AGTCAGAACCTCCAGCAACG & $165-179$ & 3 \\
\hline Stylo_26 & $(\mathrm{CA})_{14}$ & VIC & AACACTGCTGGTTAAAGGCG & TCACGCTTCTCGAAAGACAG & 179-203 & 6 \\
\hline Stylo_32 & $(\mathrm{TC})_{10}$ & NED & AAAGACCAAAGCAATGCGAG & АСТTTCTTTAACCGCCCCAC & 197-209 & 7 \\
\hline Stylo_39 & $(\mathrm{TC})_{19}$ & 6-FAM & GCCAAGGGATCACTGACTTC & AAAATCCAGCGGTGTTATCG & $232-244$ & 8 \\
\hline Stylo_41 & $(\mathrm{TA})_{10}$ & 6-FAM & CGTCTGTTGAGACATACCCG & ATTCTGGCAGTTGCCTAACG & $254-258$ & 4 \\
\hline Stylo_43 & $(\mathrm{GA})_{12}$ & 6-FAM & GACTCACAAGGCAGCTGGAG & TTCGACTTCCAACCAAAAGG & $253-263$ & 7 \\
\hline Stylo_45 & $(\mathrm{AT})_{12}$ & PET & TGATCCTTTTCAACAGTGCG & TAACCTTGCTGATCTCTGCG & $260-290$ & 15 \\
\hline Stylo_48 & $(\mathrm{TA})_{13}$ & PET & ATGAGCCAAGGCCTACTTTG & CATCCTGAACAACACATGCC & $261-283$ & 8 \\
\hline Stylo_55 & $(\mathrm{CA})_{11}$ & VIC & ACCCACGCATAGTTGAAAGC & TTCTGCACCAATACCAAGGG & $286-302$ & 8 \\
\hline Stylo_59 & $(\mathrm{TC})_{11}$ & NED & CACACTAGCCATGAGACCCC & TGACCTCAGGAAAGGACCTG & $284-288$ & 3 \\
\hline Stylo_72 & $(\mathrm{TACA})_{11}$ & 6-FAM & CTCGGTTCTTCGCACTTAGG & AACGTATGTATGGACGCACG & $89-137$ & 12 \\
\hline Stylo_73 & $(\text { CATA })_{10}$ & 6-FAM & ACGCACTCACGCACTGATAC & TGCCTTTTATCGACCTACGTG & $154-226$ & 12 \\
\hline Stylo_78 & $(\mathrm{ATGT})_{10}$ & PET & GTATTGGCCCAGTGTTCCAC & GCCATTCACTTACGAATGCAG & $165-177$ & 4 \\
\hline Stylo_80 & $(\mathrm{AGAT})_{10}$ & VIC & GCAAAGCGCAACCAATAAAC & TAGCGCTATCTACTGCGAGG & $190-214$ & 9 \\
\hline Stylo_82 & $(\mathrm{ACAT})_{19}$ & 6-FAM & GGCATTTTGTGCACTCTTTG & TCCCCATTTTCATCAAGCTC & $228-240$ & 9 \\
\hline Stylo_95 & $(\mathrm{GATG})_{10}$ & VIC & TAATACCCAGTGCCCCAATC & CTGAAAAGCTTCATGGGGAG & $284-308$ & 7 \\
\hline
\end{tabular}


Table 2 Statistical analyses in a population of 24 individuals of Stylophora sp. in the Red Sea using 17 microsatellite loci

\begin{tabular}{lrrllllc}
\hline Locus & $\mathrm{N}$ & $\mathrm{N}_{\mathrm{A}}$ & $\mathrm{Ne}$ & $\mathrm{Ho}$ & $\mathrm{He}$ & $\mathrm{uHe}$ & \multicolumn{1}{l}{ Fis } \\
\hline Stylo_17 & 24 & 8 & 2.636 & 0.5 & 0.621 & 0.634 & 0.194 \\
Stylo_19 & 21 & 3 & 1.96 & 0.429 & 0.49 & 0.502 & 0.125 \\
Stylo_26* & 22 & 6 & 3.781 & 0.364 & 0.736 & 0.753 & 0.506 \\
Stylo_32 & 21 & 7 & 3.419 & 0.667 & 0.707 & 0.725 & 0.058 \\
Stylo_39* & 24 & 8 & 4.056 & 0.292 & 0.753 & 0.77 & 0.613 \\
Stylo_41 & 24 & 4 & 2.039 & 0.5 & 0.51 & 0.52 & 0.019 \\
Stylo_43 & 24 & 7 & 3.918 & 0.583 & 0.745 & 0.761 & 0.217 \\
Stylo_45 & 24 & 15 & 8.409 & 0.875 & 0.881 & 0.9 & 0.007 \\
Stylo_48 & 24 & 8 & 5.284 & 0.875 & 0.811 & 0.828 & -0.08 \\
Stylo_55+ & 22 & 8 & 5.831 & 0.591 & 0.829 & 0.848 & 0.287 \\
Stylo_59 & 24 & 3 & 2.395 & 0.625 & 0.582 & 0.595 & -0.07 \\
Stylo_72* & 24 & 12 & 9.216 & 0.667 & 0.891 & 0.91 & 0.252 \\
Stylo_73+ & 24 & 12 & 6.365 & 0.625 & 0.843 & 0.861 & 0.258 \\
Stylo_78 & 24 & 4 & 2.554 & 0.75 & 0.609 & 0.621 & -0.23 \\
Stylo_80 & 24 & 9 & 3.728 & 0.792 & 0.732 & 0.747 & -0.08 \\
Stylo_82 & 20 & 9 & 2.867 & 0.5 & 0.651 & 0.668 & 0.232 \\
Stylo_95* & 23 & 7 & 4.876 & 0.565 & 0.795 & 0.813 & 0.289 \\
\hline Lociwith & & & & & &
\end{tabular}

Loci with significant deviation from HWE at alpha $=0.05$ are labeled with $(+)$ and loci with significant HWE deviations after Bonferroni correction $(* P<0.001)$ are labeled in italics and an asterisk. $N$ sample size, $N A$ number of alleles, $N e$ effective number of alleles, Fis Fixation index, Ho observed heterozygosity, $\mathrm{He}$ expected heterozygosity, $u H e$ unbiased expected heterozygosity

Acknowledgments This research was funded by King Abdullah University of Science and Technology (KAUST), Saudi Arabia. The authors wish to thank Jessica Bouwmeester for sample collection and the Bioscience Core Lab at KAUST.

Open Access This article is distributed under the terms of the Creative Commons Attribution License which permits any use, distribution, and reproduction in any medium, provided the original author(s) and the source are credited.

\section{References}

Ayre DJ, Hughes TP (2000) Geneotypic diversity and gene flow in brooding and spawning corals along the Great Barrier Reef, Australia. Evolution 54:1590-1605
Benson G (1999) Tandem repeats finder: a program to analyze DNA sequences. Nucleic Acids Res 27:573-580

Carpenter KE, Barber PH, Crandall E, Ablan-Lagman MC, Ambariyanto A et al (2011) Comparative phylogeography of the coral triangle and implications for marine management. J Mar Biol 2011:14

Douek J, Amar KO, Rinkevich B (2011) Maternal-larval population genetic traits in Stylophora pistillata, a hermaphroditic brooding coral species. Genetica 139:1531-1542

Excoffier L, Lischer H (2010) ARLEQUIN suite ver 3.5: a new series of programs to perform population genetics analyses under Linux and Windows. Mol Ecol Res 10:564-567

Flot JF, Blanchot J, Charpy L, Cruaud C, Licuanan WY et al (2011) Incongruence between morphotypes and genetically delimited species in the coral genus Stylophora: phenotypic plasticity, morphological convergence, morphological stasis or interspecific hybridization. BMC Ecol 11:22

Nishikawa A, Katoh M, Sakai K (2003) Larval settlement rates and gene flow of broadcast-spawning (Acropora tenuis) and planulabrooding (Stylophora pistillata) corals. Mar Ecol Prog Ser 256:87-97

Obura D (2012) The diversity and biogeography of western Indian Ocean reef-building corals. PLoS ONE 7(9):e45013

Peakall R, Smouse P (2012) GenAlEx 6.5: genetic analysis in excel. Population genetic software for teaching and research-an update. Bioinformatics 28:2537-2539

Rozen S, Skaletsky H (2000) Primer3 on the WWW for general users and for biologist programmers. Methods Mol Biol 132:365-386

Stefani F, Benzoni F, Yang SY, Pichon M, Galli P, Chen CA (2011) Comparison of morphological and genetic analyses reveals cryptic divergence and morphological plasticity in Stylophora (Cnidaria, Scleractinia). Coral Reefs 30:1033-1049

Takabayashi M, Carter D, Lopez J, Hoegh-Guldberg O (2003) Genetic variation of the scleractinian coral Stylophora pistillata from western Pacific reefs. Coral Reefs 22:17-22

Van Oosterhout C, Hutchinson WF, Wills DPM, Shipley P (2004) MICRO-CHECKER: software for identifying and correcting genotyping errors in microsatellite data. Mol Ecol Notes 4:535-538

Veron JEN (2000) Corals of the world. Australian Institute of Marine Science and CRR Qld Pty Ltd, Townsville MC

Zvuloni A, Mokady O, Al-Zibdah M, Bernardi G, Gaines SD, Abelson A (2008) Local scale genetic structure in coral populations: a signature of selection. Mar Pollut Bull 56:430-438 